\title{
The nuclear localization of SWI/SNF proteins is subjected to oxygen regulation
}

\author{
Ranita Ghosh Dastidar ${ }^{\dagger}$, Jagmohan Hooda ${ }^{\dagger}$, Ajit Shah $^{\dagger}$, Thai M Cao, Robert Michael Henke and Li Zhang ${ }^{*}$
}

\begin{abstract}
Background: Hypoxia is associated with many disease conditions in humans, such as cancer, stroke and traumatic injuries. Hypoxia elicits broad molecular and cellular changes in diverse eukaryotes. Our recent studies suggest that one likely mechanism mediating such broad changes is through changes in the cellular localization of important regulatory proteins. Particularly, we have found that over 120 nuclear proteins with important functions ranging from transcriptional regulation to RNA processing exhibit altered cellular locations under hypoxia. In this report, we describe further experiments to identify and evaluate the role of nuclear protein relocalization in mediating hypoxia responses in yeast.

Results: To identify regulatory proteins that play a causal role in mediating hypoxia responses, we characterized the time courses of relocalization of hypoxia-altered nuclear proteins in response to hypoxia and reoxygenation. We found that 17 nuclear proteins relocalized in a significantly shorter time period in response to both hypoxia and reoxygenation. Particularly, several components of the SWI/SNF complex were fast responders, and analysis of gene expression data show that many targets of the SWI/SNF proteins are oxygen regulated. Furthermore, confocal fluorescent live cell imaging showed that over $95 \%$ of hypoxia-altered SWI/SNF proteins accumulated in the cytosol in hypoxic cells, while over 95\% of the proteins were nuclear in normoxic cells, as expected.
\end{abstract}

Conclusions: SWI/SNF proteins relocalize in response to hypoxia and reoxygenation in a quick manner, and their relocalization likely accounts for, in part or in whole, oxygen regulation of many SWI/SNF target genes.

Keywords: Hypoxia response, Oxygen regulation, SWI/SNF, Live cell imaging, Protein localization

\section{Background}

Living organisms ranging from yeast to mammals use oxygen to generate their cellular energy supply and to synthesize important biomolecules. Hence, they need to respond effectively to changes in oxygen levels in the environment, particularly to hypoxia [1,2]. In humans, hypoxia is responsible for death or damage by the ischemia accompanying heart attack, stroke, and traumatic injuries [3-5]. The molecular and cellular events induced by changes in oxygen levels are very broad in eukaryotes. For example, over $20 \%$ of yeast genes change their transcript levels in response to hypoxia [6]. In the human arterial endothelial cells, more than $8 \%$ of all genes alter their transcript levels by at least 1.5 -fold in response to hypoxia [7]. In the human

\footnotetext{
* Correspondence: li.zhang@utdallas.edu

${ }^{\dagger}$ Equal contributors

Department of Molecular and Cell Biology, Center for Systems Biology, University of Texas at Dallas, Mail Stop RL11 800 W Campbell Road,
} Richardson, TX 75080, USA primary astrocytes, more than $5 \%$ of the genes alter their transcript levels by at least 2-fold in response to hypoxia [8]. Such broad changes in gene expression likely involve coordinated actions of multiple pathways and regulators.

Previous studies have identified several transcriptional regulators, including Mga1 and Rox1, that can mediate oxygen regulation of gene expression in the yeast Saccharomyces cerevisiae $[9,10]$. However, these regulators can account for the regulation of only a fraction of hypoxia-regulated genes [6]. Many other regulators are likely involved in mediating oxygen regulation. Recently, in an effort to systematically identify proteins that can mediate oxygen regulation and signaling, we performed a genomewide screen for proteins that exhibit altered cellular distribution patterns in response to hypoxia and reoxygenation [11]. We found that over 200 proteins alter their cellular locations in response to hypoxia. Particularly, under hypoxia, a good number (at least 121) of nuclear proteins do not localize to the nucleus, but accumulate in the cytosol.

\section{Biomed Central}

(c) 2012 Ghosh Dastidar et al.; licensee BioMed Central Ltd. This is an Open Access article distributed under the terms of the Creative Commons Attribution License (http://creativecommons.org/licenses/by/2.0), which permits unrestricted use, distribution, and reproduction in any medium, provided the original work is properly cited. 
In response to reoxygenation, they readily localize to the nucleus. Notably, many of these hypoxia-redistributed nuclear proteins are subunits of key regulatory complexes involved in chromatin remodeling (such as the SWI/SNF complex) [12-14], in transcriptional regulation (such as the SAGA complex) [15], and in splicing (such as the MRP complex) [16]. Hence, it is conceivable that some of these complexes can play a dominant role in mediating oxygen regulation of gene expression.

To further assess the roles of these regulators in mediating oxygen signaling and regulation, we examined the time course characteristics of the relocalization of these proteins in response to hypoxia and reoxygenation. We found a small group of nuclear proteins relocalized in a significantly shorter time period in response to both hypoxia and reoxygenation, when compared to other proteins. These proteins include three components of the SWI/SNF complex. Furthermore, using confocal fluorescent imaging of live cells, we quantitatively characterized the effect of hypoxia on the distribution of SWI/SNF proteins. We found that in live hypoxic cells, over 95\% of Swi3, Snf5, Snf6, Snf11, Snf12 and Swp82 were in the cytosol, while over $95 \%$ of hypoxiaunaffected proteins, such as Swi2 and Taf14, were in the nucleus. These results suggest that hypoxia can significantly alter the composition and property of the SWI/SNF complex and mediate oxygen regulation of gene expression.

\section{Results}

Among the hypoxia-redistributed nuclear proteins we previously identified, some are likely involved in mediating oxygen signaling and regulation of gene expression. Particularly, proteins that change their locations in relatively shorter time periods are likely the regulators that initiate further downstream events in responses to hypoxia and reoxygenation. In other words, they are likely to be positioned in the upstream of the hierarchy of the molecular events elicited by hypoxia or reoxygenation, and are responsible for initiating downstream changes such as those in gene expression. We therefore decided to characterize the time course response of the hypoxia-redistributed nuclear proteins in response to hypoxia and reoxygenation.

First, we examined the time course characteristics of nuclear proteins in response to hypoxia. We found that all proteins became predominantly cytosolic after exposure to hypoxia for 12 hours; see Snf11 in Figure 1A for an example. One group of these proteins became predominantly cytosolic after only 6 hours or shorter times; see Swp82 in Figure 1A for an example. This group has 48 proteins (see Table 1 ). They include several transcriptional regulators and regulators of chromatin, DNA replication and repair, and RNA processing (Figure 2). Notably, five components of the SWI/SNF complex relocalized in 6 hours (see Table 1 and Figure 2), suggesting that they may have a signaling role in initiating downstream events.

Second, we characterized the changes in protein distribution when cells grown under hypoxia were exposed to oxygen. We found that 76 hypoxia-redistributed nuclear proteins (see Table 2) recovered their nuclear locations in the majority of cells in one hour; see Swi3 in Figure $1 \mathrm{~B}$ for an example. The rest of the proteins recovered their nuclear location in the majority of the cells in 2 or more hours; see Snf5 in Figure 1B for an example. Among these nuclear proteins, 17 of them responded to both hypoxia and reoxygenation in shorter times than the rest of the proteins (see Figure 3). Notably, 3 of these faster responding proteins are components
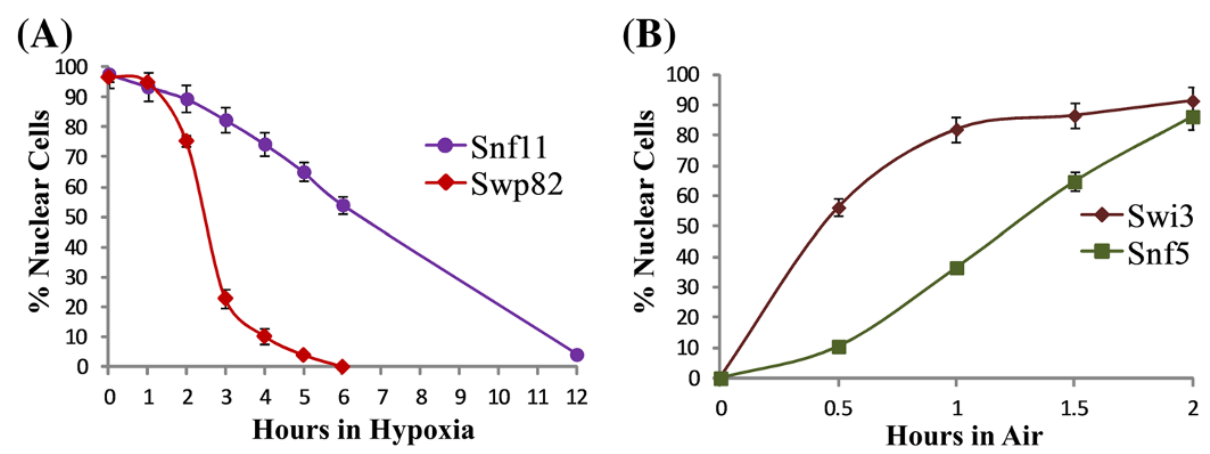

Figure 1 Time course characteristics of protein relocalization elicited by hypoxia or reoxygenation. (A) The time courses of relocalization of Snf11 and Swp82 in response to hypoxia. Cells expressing Snf11-GFP or Swp82-GFP were grown in air and then shifted to hypoxic growth conditions. At various time points, cells were imaged, and the number of cells showing GFP-tagged proteins in the nucleus (N) or cytosol (C) was counted. The percentage of cells showing nuclear locations is calculated and plotted. (B) The time courses of relocalization of Snf5 and Swi3 in response to reoxygenation. Cells expressing Snf5-GFP or Swi3-GFP were grown under hypoxia and then shifted to normoxic growth conditions. At various time points, cells were imaged, and the number of cells showing GFP-tagged proteins in the nucleus (N) or cytosol (C) was counted. The percentage of cells showing nuclear localization is plotted here. 
Table 1 Nuclear proteins that relocalized to the cytosol in response to hypoxia in a shorter time period

\begin{tabular}{|c|c|c|}
\hline ORF name & Gene name & Description \\
\hline YOR113W & AZF1 & Involved in glucose induction of CLN3 transcription \\
\hline YML102W & CAC2 & Component of the chromatin assembly complex \\
\hline YKL022C & CDC16 & Subunit of the anaphase-promoting complex/cyclosome \\
\hline YFR036W & CDC26 & Subunit of the Anaphase-Promoting Complex/Cyclosome \\
\hline YIL036W & CST6 & Member of the ATF/CREB family \\
\hline YML113W & DAT1 & DNA binding protein that recognizes oligo(dA).oligo(dT) tracts \\
\hline YIL131C & $\mathrm{FKH1}$ & Forkhead family transcription factor \\
\hline YNL068C & $\mathrm{FKH} 2$ & Forkhead family transcription factor \\
\hline YDR096W & GIS1 & JmjC domain-containing histone demethylase \\
\hline YDR295C & HDA2 & Subunit of a class II histone deacetylase complex \\
\hline YPR179C & HDA3 & Subunit of a class II histone deacetylase complex \\
\hline YOR038C & HIR2 & Subunit of the HIR nucleosome assembly complex \\
\hline YDL108W & KIN28 & Subunit of the transcription factor TFIIH \\
\hline YNR024W & MPP6 & RNA binding protein that associates with the exosome \\
\hline YGL013C & PDR1 & Master regulator of multidrug resistance genes \\
\hline YDL106C & $\mathrm{PHO} 2$ & Homeobox transcription factor \\
\hline YJR006W & POL31 & DNA polymerase III (delta) subunit \\
\hline YNL282W & POP3 & Subunit of both RNase MRP \\
\hline YBL018C & POP8 & Subunit of both RNase MRP \\
\hline YKL113C & RAD27 & 5' to $3^{\prime}$ exonuclease, 5' flap endonuclease \\
\hline YPL153C & RAD53 & Required for cell-cycle arrest in response to DNA damage \\
\hline YMR182C & RGM1 & Putative transcriptional repressor \\
\hline YBR095C & RXT2 & Subunit of the histone deacetylase Rpd3L complex \\
\hline YDR180W & SCC2 & Subunit of cohesin loading factor ( $S c c 2 p-S c c 4 p)$ \\
\hline YGL066W & SGF73 & Subunit of SAGA histone acetyltransferase complex \\
\hline YIL104C & SHQ1 & Required for the assembly of box H/ACA snoRNPs \\
\hline YHR206W & SKN7 & Regulator for optimal induction of heat-shock genes \\
\hline YDR073W & SNF11 & Subunit of the SWI/SNF chromatin remodeling complex \\
\hline YNR023W & SNF12 & $73 \mathrm{kDa}$ subunit of the SWI/SNF chromatin remodeling complex \\
\hline YBR289W & SNF5 & Subunit of the SWI/SNF chromatin remodeling complex \\
\hline YHL025W & SNF6 & Subunit of the SWI/SNF chromatin remodeling complex \\
\hline YCR033W & SNT1 & Subunit of the Set $3 \mathrm{C}$ deacetylase complex \\
\hline YPL138C & SPP1 & Subunit of the COMPASS complex \\
\hline YBR152W & SPP381 & Component of U4/U6.U5 tri-snRNP \\
\hline YDR464W & SPP41 & Negative regulator of expression of PRP4 and PRP3 \\
\hline YDR392W & SPT3 & Subunit of the SAGA and SAGA complexes \\
\hline YJL176C & SWI3 & Subunit of the SWI/SNF chromatin remodeling complex \\
\hline YFL049W & SWP82 & Subunit of the SWI/SNF chromatin remodeling complex \\
\hline YDR334W & SWR1 & Component of the SWR1 complex \\
\hline YDR416W & SYF1 & Component of the spliceosome complex \\
\hline YDR079C-A & TFB5 & Component of TFIIH \\
\hline YNL273W & TOF1 & Subunit of a replication-pausing checkpoint complex \\
\hline YPL203W & TPK2 & cAMP-dependent protein kinase catalytic subunit \\
\hline YBR030W & YBR030W & Putative ribosomal lysine methyltransferase \\
\hline
\end{tabular}


Table 1 Nuclear proteins that relocalized to the cytosol in response to hypoxia in a shorter time period (Continued)

\begin{tabular}{lll}
\hline YGR093W & YGR093W & Putative debranching enzyme associated ribonuclease \\
YLR455W & YLR455W & Putative protein of unknown function \\
YNL035C & YNL035C & Putative protein of unknown function \\
YPR107C & YTH1 & Component of cleavage and polyadenylation factor \\
\hline
\end{tabular}

of the SWI/SNF complex (Figure 3). These and previous results strongly suggest that the SWI/SNF proteins play regulatory roles in mediating oxygen regulation and hypoxia response. Given their roles in chromatin remodeling and transcriptional regulation $[17,18]$, they are likely responsible for initiating certain changes in gene expression in response to changes in oxygen levels. Although 14 other proteins also responded to hypoxia and reoxygenation in shorter times, they are generally not components of one regulatory complex (Figure 3 ).

Therefore, we decided to further characterize the effect of hypoxia on the SWI/SNF proteins. First, we examined if changes in oxygen levels affect the protein levels of SWI/ SNF proteins. To this end, we detected and compared the levels of SWI/SNF proteins in hypoxic and normoxic cells. We used yeast strains expressing the SWI/SNF proteins with the TAP tag at the C-terminus from the natural chromosomal locations [19]. We found that the levels of all detected SWI/SNF proteins were not significantly affected by hypoxia (Figure 4). The variations in the ratios of protein levels in hypoxic vs. normoxic cells were generally less than $30 \%$, suggesting that hypoxia did not cause significant degradation of the Swi/Snf proteins during the time period when the proteins would be relocalized to the cytosol. These proteins include those whose cellular location was affected by hypoxia, such as Snf6, Swi3, Swp82 and Snf11 (see Figure 4). They also include all those SWI/SNF proteins whose localization was not affected by hypoxia. These results show that the levels of SWI/SNF proteins are not regulated by oxygen levels.

Therefore, the regulation of nuclear localization is likely the dominant mechanism mediating oxygen regulation of SWI/SNF proteins and the regulation of their targets. To further confirm the regulation of nuclear localization of the SWI/SNF proteins by oxygen, we quantitatively examined and compared their distribution in live hypoxic and normoxic cells, by using confocal fluorescent live cell imaging. As expected, for the SWI/SNF proteins whose localization was not affected by oxygen levels, over $95 \%$ of the proteins was present in the nucleus

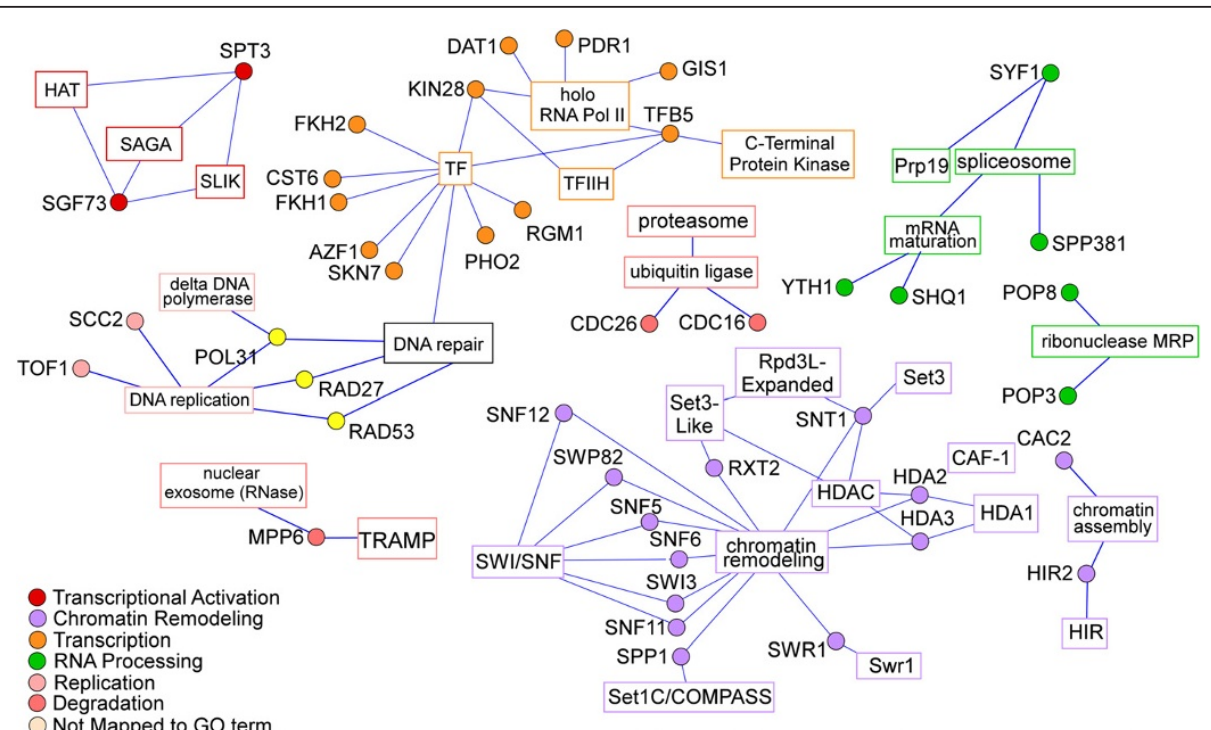

YGR093W $\bigcirc$ YLR455W $\bigcirc$ YNL035C $\bigcirc$ RKM3 $\bigcirc$ TPK2 $\bigcirc$

Figure 2 Graphical representation of protein-protein interaction networks for the nuclear proteins that localized to the cytosol in response to hypoxia in a shorter time period. The information on the biochemical interactions and complex formation of the 48 faster responding nuclear proteins (listed in Table 1) was downloaded from the SGD database, and then imported to Cytoscape for network construction. The proteins are shown as round nodes in different colors based on their cellular functions. The GO terms for protein complexes or functional categorizations are indicated and are shown in square nodes. Nodes of the same sub-networks are colored similarly, and a key for the coloring of the nodes is shown. Lines represent an association of the protein to a particular complex or functional GO term. 
Table 2 Proteins that recovered their nuclear locations in response to oxygen in a shorter time period

\begin{tabular}{|c|c|c|}
\hline ORF name & Gene name & Description \\
\hline YBR236C & $\mathrm{ABD1}$ & Methyltransferase \\
\hline YPR180W & AOS1 & Smt3p (SUMO) activator \\
\hline YJL115W & ASF1 & Nucleosome assembly factor \\
\hline YNR010W & CSE2 & Subunit of the RNA polymerase II mediator complex \\
\hline YIL036W & CST6 & Member of the ATF/CREB family \\
\hline YJL006C & CTK2 & Beta subunit of C-terminal domain kinase I \\
\hline YEL018W & EAF5 & Subunit of the NuA4 acetyltransferase complex \\
\hline YMR277W & FCP1 & Carboxy-terminal domain (CTD) phosphatase \\
\hline YCL011C & GBP2 & Poly(A+) RNA-binding protein \\
\hline YGR252W & GCN5 & Subunit of the ADA and SAGA complexes \\
\hline YDR096W & GIS1 & JmjC domain-containing histone demethylase \\
\hline YDR174W & HMO1 & Chromatin associated high mobility group family member \\
\hline YFL013C & IES1 & Subunit of the INO80 chromatin remodeling complex \\
\hline YHR085W & $|\mathrm{P}| 1$ & Essential component of the Rix 1 complex \\
\hline YIL026C & IRR1 & Subunit of the cohesin complex \\
\hline YDL108W & KIN28 & Subunit of the transcription factor TFIIH \\
\hline YDL087C & LUC7 & Associated with the U1 snRNP complex \\
\hline YMR043W & MCM1 & Involved in cell-type-specific transcription \\
\hline YDL005C & MED2 & Subunit of the RNA polymerase II mediator complex \\
\hline YMR070W & МOT3 & Nuclear transcription factor mediating hypoxia response \\
\hline YKL059C & MPE1 & Essential conserved subunit of CPF \\
\hline YNR024W & MPP6 & Nuclear RNA binding protein \\
\hline YLR116W & MSL5 & Component of the commitment complex \\
\hline YPR144C & NOC4 & Mediating maturation and nuclear export of 405 \\
\hline YHR133C & NSG1 & Regulator of sterol biosynthesis \\
\hline YKR082W & NUP133 & Subunit of the nuclear pore complex \\
\hline YAR002W & NUP60 & Subunit of the nuclear pore complex \\
\hline YJL061W & NUP82 & Nucleoporin, subunit of the nuclear pore complex (NPC) \\
\hline YOL115W & PAP2 & Catalytic subunit of TRAMP \\
\hline YDR228C & PCF11 & mRNA 3' end processing factor \\
\hline YMR076C & PDS5 & Required for sister chromatid condensation and cohesion \\
\hline YNL282W & POP3 & Subunit of both RNase MRP \\
\hline YGR030C & POP6 & Subunit of both RNase MRP \\
\hline YBL018C & POP8 & Subunit of both RNase MRP \\
\hline YLL036C & PRP19 & Splicing factor associated with the spliceosome \\
\hline YGR156W & PTI1 & Pta1p Interacting protein \\
\hline YKL113C & RAD27 & 5' to $3^{\prime}$ exonuclease, 5 ' flap endonuclease \\
\hline YGL246C & RAl1 & Required for pre-rRNA processing \\
\hline YNL216W & RAP1 & Involved in either activation or repression of transcription \\
\hline YDR195W & REF2 & RNA-binding protein \\
\hline YARO07C & RFA1 & Subunit of heterotrimeric Replication Protein A \\
\hline YNL290W & $\mathrm{RFC3}$ & Subunit of heteropentameric Replication factor C \\
\hline YOL094C & RFC4 & Subunit of heteropentameric Replication factor C \\
\hline YHR197W & RIX1 & Essential component of the Rix 1 complex \\
\hline
\end{tabular}


Table 2 Proteins that recovered their nuclear locations in response to oxygen in a shorter time period (Continued)

\begin{tabular}{|c|c|c|}
\hline YMR061W & RNA14 & Cleavage and polyadenylation factor I (CF I) component \\
\hline YJL011C & RPC17 & RNA polymerase III subunit C17 \\
\hline YER117W & RPL23B & Component of the large (60S) ribosomal subunit \\
\hline YDR427W & RPN9 & Non-ATPase regulatory subunit of the 265 proteasome \\
\hline YHR062C & RPP1 & Subunit of both RNase MRP \\
\hline YBR095C & RXT2 & Subunit of the histone deacetylase Rpd3L complex \\
\hline YIL084C & SDS3 & Component of the Rpd3p/Sin3p deacetylase complex \\
\hline YJL168C & SET2 & Histone methyltransferase \\
\hline YIL104C & SHQ1 & Required for the assembly of box H/ACA snoRNPs \\
\hline YHR206W & SKN7 & Regulator of heat-shock genes \\
\hline YGR074W & SMD1 & Core Sm protein Sm D1 \\
\hline YHL025W & SNF6 & Subunit of the SWI/SNF chromatin remodeling complex \\
\hline YMR016C & SOK2 & Involved in the CAMP-dependent protein kinase signaling \\
\hline YBR152W & SPP381 & mRNA splicing factor \\
\hline YER161C & SPT2 & Involved in negative regulation of transcription \\
\hline YDR392W & SPT3 & Subunit of the SAGA and SAGA-like complexes \\
\hline YIL143C & SSL2 & Component of RNA polymerase transcription factor TFIIH \\
\hline YBR231C & SWC5 & Component of the SWR1 complex \\
\hline YJL176C & SWI3 & Subunit of the SWI/SNF chromatin remodeling complex \\
\hline YFL049W & SWP82 & Subunit of the SWI/SNF chromatin remodeling complex \\
\hline YGR129W & SYF2 & Component of the spliceosome complex \\
\hline YGR274C & TAF1 & TFIID subunit (145 kDa) \\
\hline YGL112C & TAF6 & Subunit (60 kDa) of TFIID and SAGA complexes \\
\hline YDR311W & TFB1 & Subunit of TFIIH and nucleotide excision repair factor complexes \\
\hline YPL203W & TPK2 & CAMP-dependent protein kinase catalytic subunit \\
\hline YDR165W & TRM82 & Subunit of a tRNA methyltransferase complex \\
\hline YNL246W & VPS75 & NAP family histone chaperone \\
\hline YOR229W & WTM2 & Regulator of meiosis, silencing, and expression of RNR genes \\
\hline YHR090C & YNG2 & Subunit of the NuA4 histone acetyltransferase complex \\
\hline YIL063C & YRB2 & Involved in nuclear processes of the Ran-GTPase cycle \\
\hline YGR270W & YTA7 & Regulator of histone gene expression \\
\hline YPR107C & YTH1 & Component of cleavage and polyadenylation factor \\
\hline
\end{tabular}

in both normoxic and hypoxic cells (see Figure 5). Figure 5A shows the distribution of Taf14 in air and under hypoxia, while Figure 5B shows the distribution of Swi2. For those proteins whose localization was affected by oxygen, over $95 \%$ of the proteins was present in the nucleus in air, whereas over $95 \%$ of the proteins was present in the cytosol under hypoxia (Figures 6 and 7). Figure 6A shows the distribution of Swi3 in normoxic cells, while Figure 6B shows the distribution of Swi3 in hypoxic cells. We also quantified the distribution of other hypoxia-relocalized SWI/SNF proteins (Figure 7). Figure 7A-E show the distribution of Snf5, Snf6, Snf11, Snf12 and Swp82 in hypoxic cells (The images for normoxic cells invariably showed nuclear localization, as expected and as shown in Figures 5 and 6, and are therefore omitted). Clearly, Swi2, Snf5, Snf6, Snf11, Snf12 and Swp82 proteins were transported to the nucleus in normoxic cells, but they accumulated in the cytosol in hypoxic cells.

To further ascertain the role of SWI/SNF proteins in oxygen regulation of gene expression, we determined if and how many oxygen-regulated genes are SWI/SNF protein targets as well. To this end, we used our previous microarray and computational work analyzing genes regulated by oxygen/ and $\Delta$ hap 1 cells [6]. We also used the previously identified targets of 263 transcription factors [20]. Using these two sets of data and the $\mathrm{R}$ program, we identified those hypoxia-regulated genes that are targets of SWI/SNF proteins and calculated the p- 


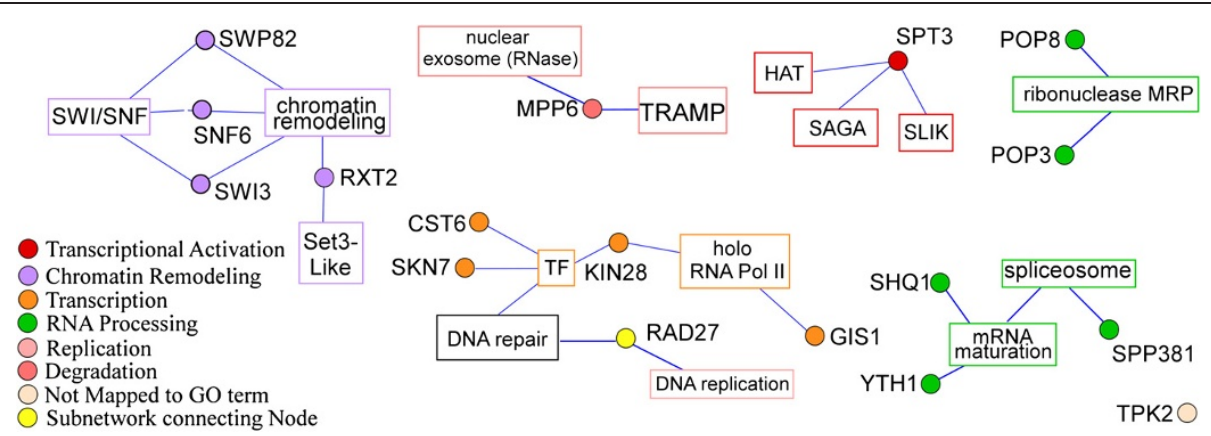

Figure 3 Graphical representation of protein-protein interaction networks for the nuclear proteins that changed their locations in response to hypoxia and reoxygenation in shorter time periods. The information on the biochemical interactions and complex formation of the 17 faster responding nuclear proteins was downloaded from the SGD database, and then imported to Cytoscape for network construction. The proteins are shown as round nodes in different colors based on their cellular functions. The GO terms for protein complexes or functional categorizations are indicated and shown in square nodes. Nodes of the same sub-networks are colored similarly, and a key for the coloring of the nodes is shown. Lines represent an association of the protein to a particular complex or functional GO term.

values. Table 3 shows that in the wild type HAP1 cells, 95, 112, 67, 109, 9 and 19 target genes of Swi2, Swi3, Snf5, Snf6, Snf11 and Taf14, respectively, are oxygen regulated. In $\Delta$ hap1 cells, similar numbers of these SWI/SNF targets are hypoxia altered. These results strongly suggest that SWI/SNF proteins play a major role in mediating oxygen regulation and hypoxia responses. Furthermore, the changes in the relocalization of SWI/SNF proteins in response to hypoxia are completed between 6-12 hours or 1-2 generations; and the changes in the relocalization of SWI/SNF proteins in response to reoxygenation are completed in less than one generation. In contrast, the transcriptome response to hypoxia are completed after 5-6 generations; and the transcriptome response to reoxygenation are completed in 2 generations [21]. These results show that changes in SWI/SNF protein localization precede transcriptome responses. They therefore strongly suggest that oxygen regulation of SWI/SNF protein localization contribute to, at least in part, oxygen regulation of gene expression.
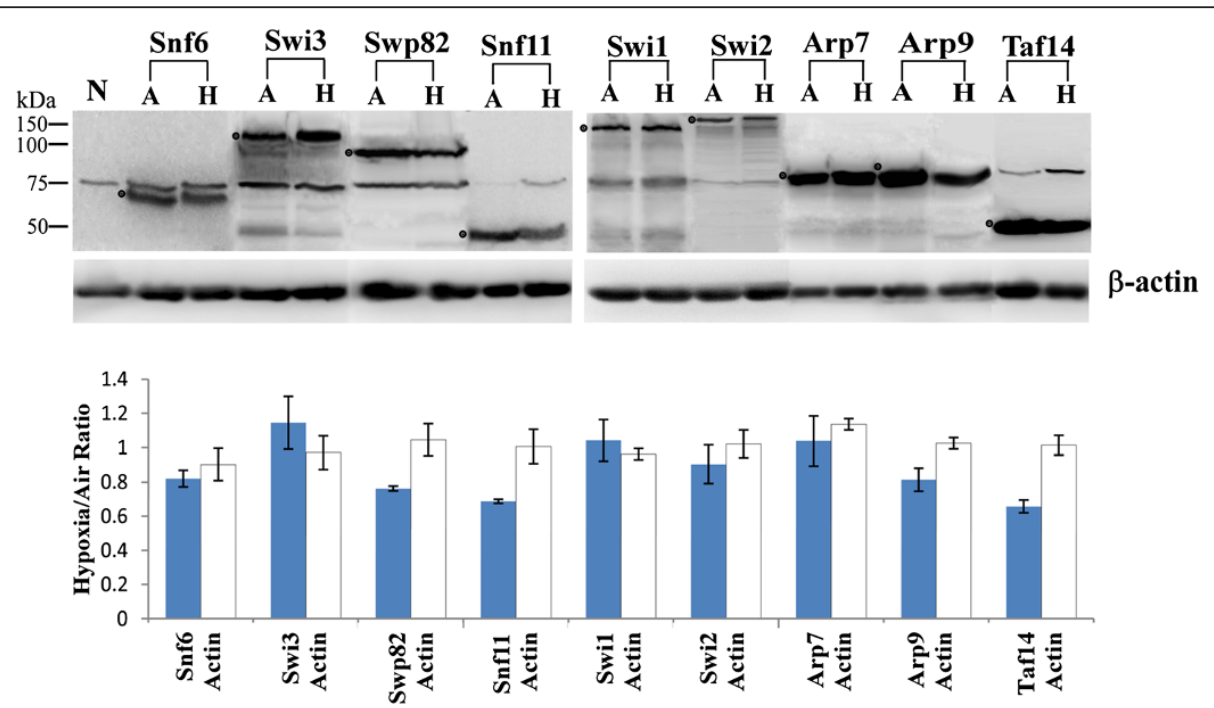

Figures 4 Western blot showing TAP-tagged proteins in extracts prepared from normoxic and hypoxic cells. Shown here are proteins in extracts from the parent BY4741 cells without any TAP-tagged proteins expressed (N), and from cells grown in air (A) or under hypoxia (H) which expresses Snf6-TAP (molecular mass: 58 kDa), Swi3-TAP (113 kDa), Swp82-TAP (90 kDa), Snf11-TAP (40 kDa), Swi1-TAP (168 kDa), Swi2-TAP (214 kDa), Arp7-TAP (74 kDa), Arp9-TAP (73 kDa), Taf14-TAP (47 kDa), respectively. For the hypoxic condition, cells were placed in a hypoxia chamber for up to 12 hours (the time period necessary for the proteins to relocate to the cytosol). The intensity of bands representing the Swi/Snf proteins was quantified, and the intensity ratios of the bands representing the Swi/Snf proteins in hypoxic vs. normoxic cells were plotted and shown below the Western blot images. The data plotted are averages of three replicates. 


\section{(A) Taf14}

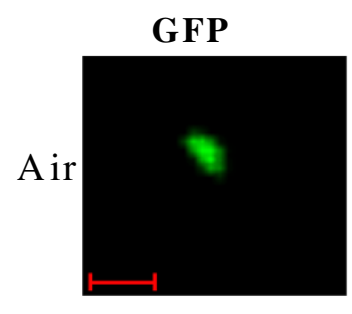

\section{DAPI}

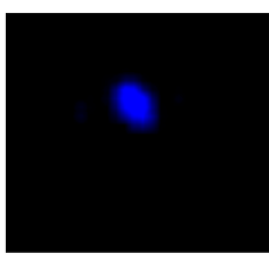

DAPI
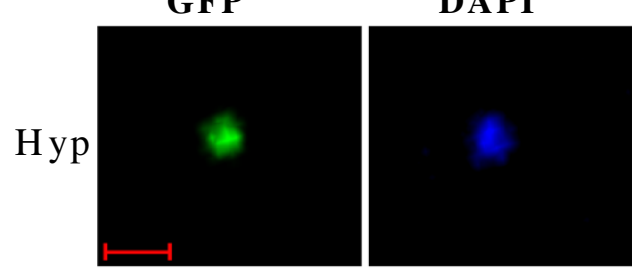

(B) $\mathrm{S}$ w i2
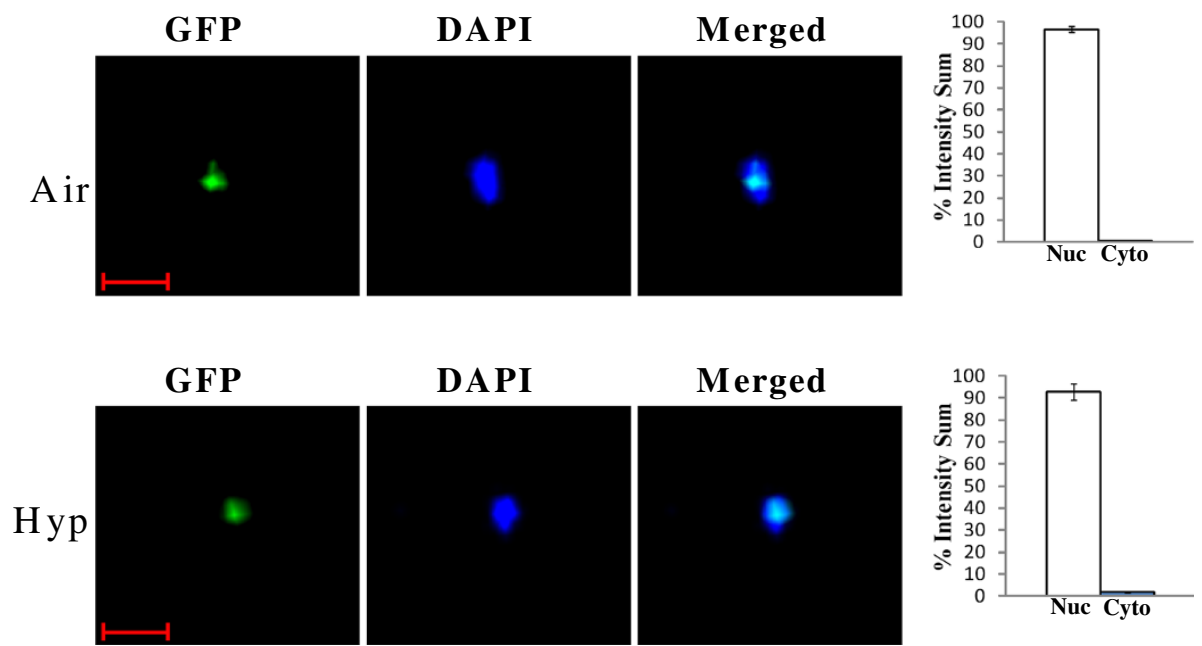

Figure 5 Examples of GFP, DAPI and merged confocal fluorescent images of cells expressing proteins whose cellular localization is not affected by hypoxia. Cells expressing Taf14-GFP (A) or Swi2-GFP (B) were grown in air or under hypoxia (Hyp), and the images were captured. The percentages of GFP fluorescence in the nucleus (N) or cytosol (C) were quantified and plotted here. The scale bar represents $1 \mu \mathrm{m}$.

\section{Discussion}

The SWI/SNF complex is an ATP-dependent chromatin remodeling complex [22]. Its composition and function are conserved from yeast to humans [13]. In yeast, more than $10 \%$ of the genes are the targets of SWI/SNF proteins, although the targets of different SWI/SNF proteins are different [20]. Hypoxia and reoxygenation induce changes in gene expression in over $20 \%$ of yeast genes [6]. Such broad changes in gene expression involve the action of an array of regulators. Previous studies have shown that Mga2, Rox1, Hap1 and Mot3 are all involved in mediating oxygen regulation of several subsets of genes $[6,21,23,24]$. In this report, we show that several SWI/SNF proteins alter their subcellular localization readily in response to hypoxia or reoxygenation and that this change in subcellular localization likely contributes to oxygen regulation of SWI/SNF target genes.

Because the targets of SWI/SNF proteins overlap but are not identical [20], it is likely that different SWI/SNF proteins act on different groups of genes and control their expression. Here, we show that six SWI/SNF proteins accumulate in the cytosol in hypoxic cells, and relocalize to the nucleus in response to reoxygenation. Furthermore, several of the SWI/SNF proteins respond 


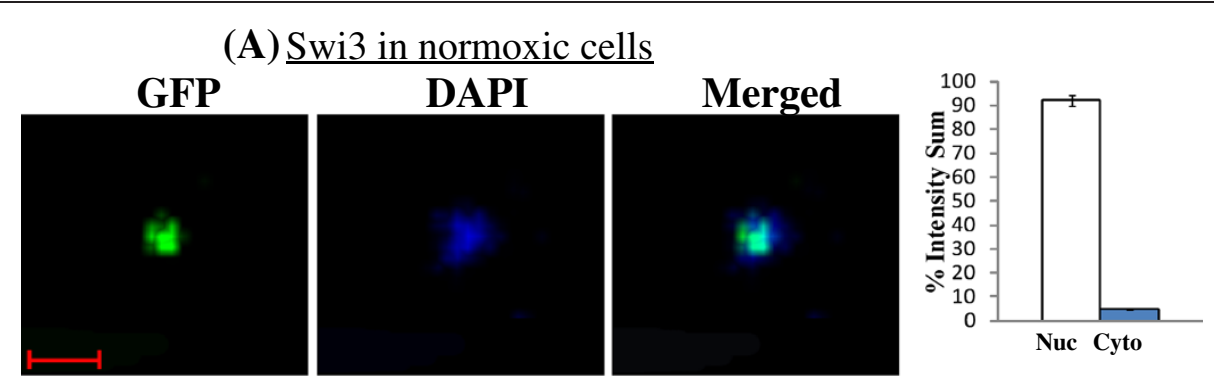

(B) $\underline{\text { Swi3 in hypoxic cells }}$
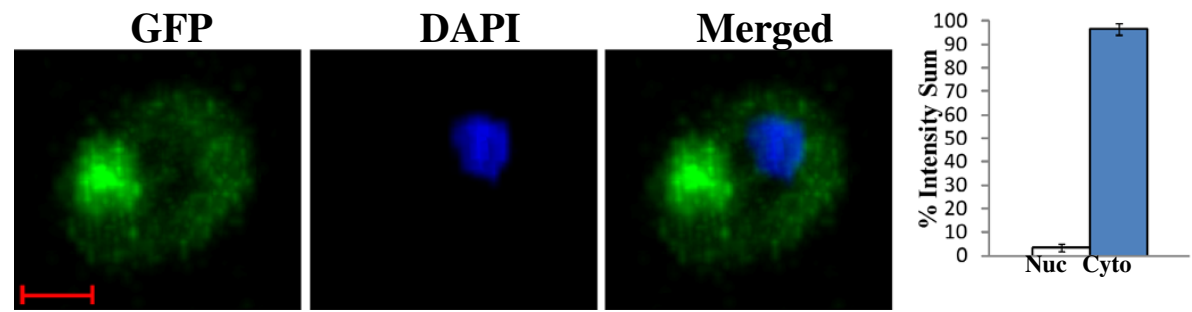

Figure 6 DAPI and merged confocal fluorescent images of cells expressing Swi3-GFP. Cells were grown in air or under hypoxia (Hyp), and the images were captured. The percentages of GFP fluorescence in the nucleus ( $\mathrm{N}$ ) or cytosol (C) was quantified and plotted here. The scale bar represents $1 \mu \mathrm{m}$.

to hypoxia or reoxygenation and relocalize in a relatively quick manner. The redistribution of the six SWI/SNF proteins in the cytosol should presumably change the composition, and thereby the function or selectivity of the SWI/SNF complexes in the nucleus. Hence, the relocalization can affect the target expression of not only these SWI/SNF proteins whose localization is altered by hypoxia, such as Swi3, but also those whose localization is not affected by hypoxia, such as Swi2 and Taf14 (Table 3). Very likely, under hypoxia, because Swi3 and other proteins are predominantly present in the cytosol, the nuclear SWI/SNF proteins, such as Swi2, are likely complexed with other proteins, and act as chromatin remodelers on different sets of target genes. This explains why many SWI/SNF target genes are altered by hypoxia/reoxygenation (Table 3). Previous studies showed that Swi2, Arp7 and Arp9 form a core subcomplex possessing the ATP-dependent remodeling activity [25], while Swi3 controls SWI/SNF assembly, ATPdependent $\mathrm{H} 2 \mathrm{~A}-\mathrm{H} 2 \mathrm{~B}$ displacement, as well as recruitment to target genes $[25,26]$. Notably, the nuclear localization of Swi2, Arp7 and Arp9 is not affected by hypoxia, while Swi3 is affected. This supports the idea that the core complex can associate with other as yet unidentified proteins and form a different kind of SWI/ SNF complexes in the nucleus in hypoxic cells.

These results suggest a model for how oxygen may modulate SWI/SNF complex composition and function (Figure 8). In normoxic cells, SWI/SNF components form complexes in the nucleus and remodel chromatin structure at their target genes. In hypoxic cells Swi3 and other five SWI/SNF proteins accumulate in the cytosol, leaving the Swi2-Arp7-Arp9 core subcomplex available to interact with other proteins. Consequently, a different kind of SWI/SNF complex containing the core complex and other proteins (A, B and C in Figure 8) can be formed and act to remodel chromatin and control gene expression in different sets of genes. In response to reoxygenation, Swi3 and other proteins can readily relocalize to the nucleus, forming the normoxic SWI/SNF complexes, and reestablish gene expression patterns under normoxic conditions.

This model is also consistent with a recent study showing that Swi3 is a key regulator in controlling respiration genes [27]. The authors used a computational approach to analyze modules of genes with a common regulation that are affected by specific DNA polymorphisms. They integrated genotypic and expression data for individuals in a segregating population with complementary expression data of strains mutated in a variety of regulatory proteins, in order to identify regulatory-linkage modules. In so doing, they found that Swi3 is a dominant regulator in the control of respiratory gene expression [27]. The effect of swi3 deletion is stronger than that of known respiratory regulators, including Hap2/3/4/5, Mot3 and Rox1. This is in complete agreement with our results showing that hundreds of SWI/SNF targets are altered by hypoxia (Table 3), and supports our model (Figure 8). The regulation of SWI/SNF protein localization may also occur in other eukaryotes. For example, in mammalian cells, recent 


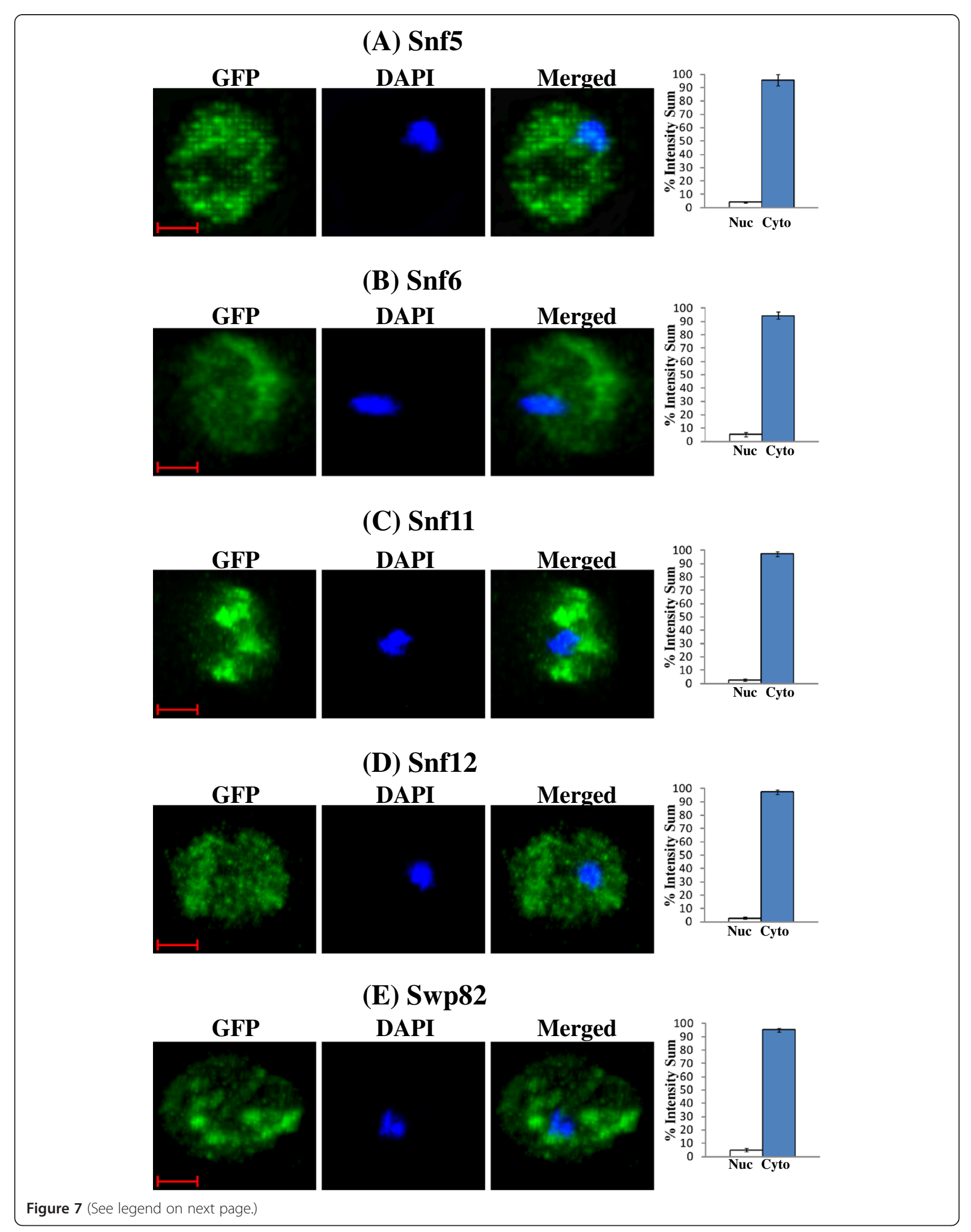


(See figure on previous page.)

Figure 7 DAPI and merged confocal fluorescent images of cells expressing SWI/SNF proteins whose cellular location is affected by hypoxia. Cells expressing Snf5-GFP, Snf6-GFP, Snf11-GFP, Snf12-GFP and Swp82-GFP were grown in air or under hypoxia (Hyp), and the images were captured. Only the images of hypoxic cells are shown, because the normoxic cells all exhibit the same nuclear pattern as shown in Figures 5 and 6 . The percentages of GFP fluorescence in the nucleus ( $N$ ) or cytosol (C) were quantified and plotted here. The scale bar represents $1 \mu \mathrm{m}$.

studies showed that SWI/SNF proteins are important for oxygen regulation in mammalian cells $[28,29]$. It is likely that SWI/SNF proteins can respond to changes in oxygen levels and regulate gene expression in diverse eukaryotes.

\section{Conclusions}

Several SWI/SNF proteins, including Swi3, Snf6 and Swp82, respond to hypoxia or reoxygenation and alter their subcellular distribution in a relatively quick manner. This change in localization likely contributes to oxygen regulation of SWI/SNF target genes.

\section{Methods}

\section{Yeast strains and antibodies}

The yeast GFP clone collection of 4159 strains expressing GFP-tagged proteins [30] was purchased from Invitrogen Corp. The anti-TAP monoclonal antibody was purchased from Open Biosystems.

\section{The creation of hypoxic growth conditions}

Hypoxic $\left(\sim 10 \mathrm{ppb} \mathrm{O}_{2}\right)$ growth condition was created by using a hypoxia chamber (Coy Laboratory, Inc.) and by filling the chamber with a mixture of $5 \% \mathrm{H}_{2}$ and $95 \% \mathrm{~N}_{2}$ in the presence of a palladium catalyst [31]. The oxygen level in the chamber was monitored by using the Model 10 gas analyzer (Coy Laboratory, Inc.). The precise level of oxygen was also estimated by using a CHEMetrics rhodazine oxygen detection kit (K-7511) with the minimum detection limit at $1 \mathrm{ppb}$, and a range of $0-20 \mathrm{ppb}$. The hypoxic state was further confirmed by measuring oxygen-controlled promoter activities, including UAS1/ $C Y C 1, A N B 1$ and OLE1 $[9,10,31]$.

Table 3 The number of Swi/Snf targets whose transcript level is regulated by oxygen

\begin{tabular}{lccccc}
\hline & \multicolumn{2}{c}{ HAP1 cells } & & \multicolumn{2}{c}{$\Delta$ hap1 cells } \\
\cline { 2 - 3 } \cline { 6 - 6 } & Targets & p-value & & Targets & p-value \\
\hline Swi2 & 95 & $7.55 \mathrm{E}-69$ & & 119 & $2.87 \mathrm{E}-69$ \\
Swi3 & 112 & $6.14 \mathrm{E}-94$ & & 118 & $7.31 \mathrm{E}-102$ \\
Snf5 & 67 & $5.24 \mathrm{E}-49$ & & 71 & $1.05 \mathrm{E}-53$ \\
Snf6 & 109 & $8.06 \mathrm{E}-73$ & & 139 & $1.10 \mathrm{E}-107$ \\
Snf11 & 9 & $6.79 \mathrm{E}-10$ & & 6 & $7.45 \mathrm{E}-06$ \\
Taf14 & 19 & $8.09 \mathrm{E}-19$ & & 20 & $3.0 \mathrm{E}-20$ \\
\hline
\end{tabular}

Time course characterization of cellular localization of SWI/SNF proteins

For a time course characterization of SWI/SNF protein relocalization in response to hypoxia or reoxygenation, we used a previously defined nuclear protein import assay in yeast [32-34]. Briefly, cells expressing GFPtagged proteins at various time points of hypoxia or reoxygenation treatment were collected, and images were acquired. At least 25 cells were counted at each time point, and three sets of cells were counted. A particular cell was counted as having the GFP-tagged protein in the nucleus if the nucleus was much brighter than the surrounding cytoplasm and a clear nuclear-cytoplasmic boundary was visible. Cells with excessive bright or weak fluorescence or with aberrant morphology were not scored.

\section{Confocal fluorescent live cell imaging and quantitation}

GFP-tagged strains were grown in synthetic complete media in air or in a hypoxia chamber. Cells were collected and subjected to confocal fluorescent imaging and quantitation. Image acquisition of live cells was performed by using a Perkin Elmer UltraView ERS Spinning Disc Confocal Microscope (Perkin Elmer, Waltham, MA) with a Zeiss 100x/1.4 Oil Immersion objective (Carl Zeiss, Thornwood, NY). High speed images were captured by using a Hamamatsu EMCCD C9100 digital camera (Hamamatsu Corporation, Bridgewater, NJ). Zstacks were recorded for the DAPI channel (EX 405nm) and the GFP channel (EX $488 \mathrm{~nm}$ ) by moving the objective turret with a UltraView z-focus drive (Perkin Elmer, Waltham, MA). Volocity 5.4.2 (Improvision, Perkin Elmer, MA, USA) was used for image acquisition. The 3D confocal images were analyzed, and statistical data were collected by using Imaris 7.4.0 (Bitplane, South Windsor, CT).

\section{Preparation of yeast cell extracts and Western blotting}

Yeast cells expressing various TAP-tagged proteins were grown to an optical density $\left(\mathrm{OD}_{600}\right)$ of approximately 0.8. Cells were harvested and resuspended in 3 packed cell volumes of buffer $\left(20 \mathrm{mM}\right.$ Tris, $10 \mathrm{mM} \mathrm{MgCl}_{2}, 1$ $\mathrm{mM}$ EDTA, $10 \%$ glycerol, $1 \mathrm{mM}$ dithiothreitol, $0.3 \mathrm{M}$ $\mathrm{NaCl}, 1 \mathrm{mM}$ phenylmethylsulfonyl fluoride, $1 \mathrm{mg}$ of pepstatin per $\mathrm{ml}, 1 \mathrm{mg}$ of leupeptin per $\mathrm{ml}$ ). Cells were then permeabilized by agitation with 4 packed cell volumes of glass beads, and extracts were collected as described 


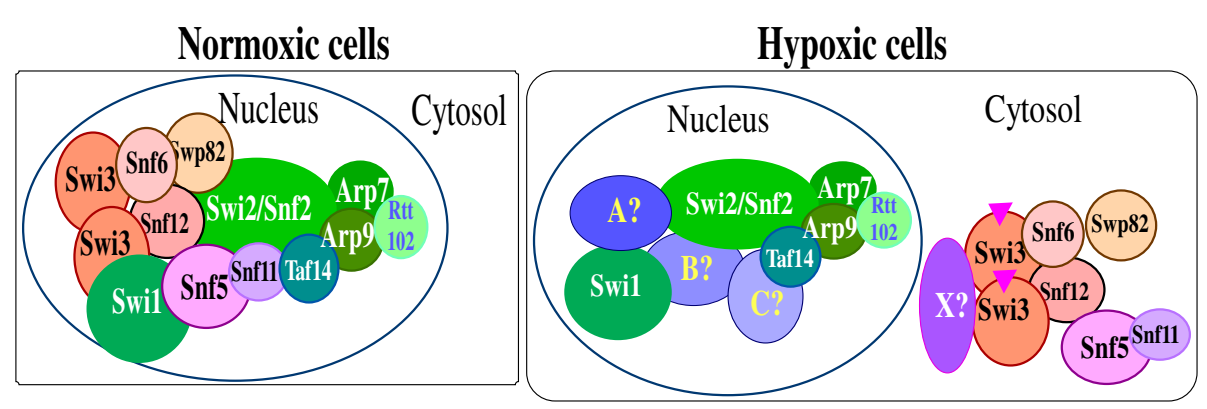

Figure 8 A cartoon illustrating how oxygen may affect SWI/SNF composition and function. In normoxic cells, the components form the SWI/SNF complex in the nucleus, enabling it to remodel chromatin at the target genes. In hypoxic cells, Swi3 and five other components are retained in the cytosol, perhaps due to modifications of these components and/or interactions with unidentified factor(s) X. In the nucleus, Swi2 and other remaining components may interact with some other proteins (marked as A, B, and C), forming complexes with different composition and targeting different sets of genes.

previously [35]. Protein concentrations were determined by the BCA (bicinchoninic acid) protein assay kit (Pierce).

For Western blotting, approximately $100 \mu \mathrm{g}$ of wholecell extracts were first separated on $8 \%$ sodium dodecyl sulfate (SDS)-polyacrylamide gels and then transferred to polyvinylidene difluoride or nitrocellulose membranes (Bio-Rad Laboratories). TAP-tagged proteins were detected by using a monoclonal antibody against TAP and a chemiluminescence Western blotting kit (Roche Diagnostics). The signals were detected and quantified by using a Kodak image station 4000MM Pro with the molecular imaging software, version 4.5.

Protein GO analysis and construction of the network map The analysis of functional categories of relocalized proteins was performed on Funspec (http://funspec.med. utoronto.ca/). For constructing the protein network map, the Cytoscape application program (http://www. cytoscape.org/) was used. The faster responding proteins identified were mapped according to their GO terms. They were obtained by using the SGD Gene Ontology Slim Mapper Web Tool set to "Macromolecular Complex terms: Components" on the SGD website. The mapped output file was reformatted into a Cytoscape compatible network file, and the network map was created. The network map was further graphically refined by using the Canvas application program. The subcellular compartments of these proteins in normoxic cells were designated based on data from the O'Shea lab [30].

\section{Competing interests}

The authors declare that they have no competing interests.

\section{Authors' contributions}

RGD, TMC and RMK performed time course studies and constructed network maps. $\mathrm{JH}$ and AS performed confocal fluorescent imaging and quantitation and analysis of regulator targets. LZ conceived of the study and drafted the manuscript. All authors read and approved the final manuscript.

\section{Acknowledgments}

This work was supported by NIH grant GM62246 (LZ). We would like to acknowledge the assistance of the UT Southwestern Live Cell Imaging Facility, a Shared Resource of the Harold C. Simmons Cancer Center, supported in part by an NCl Cancer Center Support Grant, 1P30 CA142543-01.

Received: 13 July 2012 Accepted: 17 August 2012 Published: 29 August 2012

\section{References}

1. Bunn HF, Poyton RO: Oxygen sensing and molecular adaptation to hypoxia. Physiol Rev 1996, 76:839-885.

2. Zhang H, Semenza GL: The expanding universe of hypoxia. J Mol Med 2008, 86:739-746.

3. Semenza GL: Regulation of oxygen homeostasis by hypoxia-inducible factor 1. Physiology (Bethesda) 2009, 24:97-106.

4. Johnson DR, Sherry CL, York JM, Freund GG: Acute hypoxia, diabetes, and neuroimmune dysregulation: converging mechanisms in the brain. Neuroscientist 2008, 14:235-239.

5. Simon MC, Liu L, Barnhart BC, Young RM: Hypoxia-induced signaling in the cardiovascular system. Annu Rev Physiol 2008, 70:51-71.

6. Kundaje A, Xin X, Lan C, Lianoglou S, Zhou M, Zhang L, Leslie C: A predictive model of the oxygen and heme regulatory network in yeast. PLoS Comput Biol 2008, 4:e1000224.

7. Manalo DJ, Rowan A, Lavoie T, Natarajan L, Kelly BD, Ye SQ, Garcia JG, Semenza GL: Transcriptional regulation of vascular endothelial cell responses to hypoxia by HIF-1. Blood 2005, 105:659-669.

8. Mense SM, Sengupta A, Zhou M, Lan C, Bentsman G, Volsky DJ, Zhang L: Gene expression profiling reveals the profound upregulation of hypoxiaresponsive genes in primary human astrocytes. Physiol Genomics 2006, 25:435-449.

9. Jiang Y, Vasconcelles MJ, Wretzel S, Light A, Gilooly L, McDaid K, Oh CS, Martin CE, Goldberg MA: Mga2p processing by hypoxia and unsaturated fatty acids in Saccharomyces cerevisiae: impact on LORE-dependent gene expression. Eukaryot Cell 2002, 1:481-490.

10. Lowry CV, Zitomer RS: ROX1 encodes a heme-induced repression factor regulating ANB1 and CYC7 of Saccharomyces cerevisiae. Mol Cell Biol 1988, 8:4651-4658

11. Henke RM, Dastidar RG, Shah AN, Cadinu D, Yao X, Hooda J, Zhang L: Hypoxia elicits broad and systematic changes in protein subcellular localization. Am J Physiol Cell Physiol 2011, 301:C913-C928.

12. Smith CL, Horowitz-Scherer R, Flanagan JF, Woodcock CL, Peterson CL: Structural analysis of the yeast SWI/SNF chromatin remodeling complex. Nat Struct Biol 2003, 10:141-145.

13. Mohrmann L, Verrijzer CP: Composition and functional specificity of SWI2/ SNF2 class chromatin remodeling complexes. Biochim Biophys Acta 2005 1681:59-73.

14. Peterson $\mathrm{CL}$, Workman JL: Promoter targeting and chromatin remodeling by the SWI/SNF complex. Curr Opin Genet Dev 2000, 10:187-192. 
15. Wu P-YJ, Ruhlmann C, Winston F, Schultz P: Molecular Architecture of the S. cerevisiae SAGA Complex. Molecular Cell 2004, 15:199-208.

16. Chamberlain JR, Lee Y, Lane WS, Engelke DR: Purification and characterization of the nuclear RNase P holoenzyme complex reveals extensive subunit overlap with RNase MRP. Genes Dev 1998 12:1678-1690

17. Weissman B, Knudsen KE: Hijacking the chromatin remodeling machinery: impact of SWI/SNF perturbations in cancer. Cancer Res 2009 69:8223-8230

18. Wilson BG, Roberts CW: SWI/SNF nucleosome remodellers and cancer. Nat Rev Cancer 2011, 11:481-492.

19. Ghaemmaghami S, Huh WK, Bower K, Howson RW, Belle A, Dephoure N, O'Shea EK, Weissman JS: Global analysis of protein expression in yeast. Nature 2003, 425:737-741.

20. Hu Z, Killion PJ, Iyer VR: Genetic reconstruction of a functional transcriptional regulatory network. Nat Genet 2007, 39:683-687.

21. Lai LC, Kosorukoff AL, Burke PV, Kwast KE: Metabolic-state-dependent remodeling of the transcriptome in response to anoxia and subsequent reoxygenation in Saccharomyces cerevisiae. Eukaryot Cell 2006, 5:1468-1489.

22. Clapier CR, Cairns BR: The biology of chromatin remodeling complexes. Annu Rev Biochem 2009, 78:273-304.

23. Jiang Y, Vasconcelles MJ, Wretzel S, Light A, Martin CE, Goldberg MA: MGA2 is involved in the low-oxygen response element-dependent hypoxic induction of genes in Saccharomyces cerevisiae. Mol Cell Biol 2001, 21:6161-6169.

24. Kastaniotis AJ, Zitomer RS: Rox1 mediated repression. Oxygen dependent repression in yeast. Adv Exp Med Biol 2000, 475:185-195.

25. Yang $X$, Zaurin R, Beato M, Peterson CL: Swi3p controls SWI/SNF assembly and ATP-dependent H2A-H2B displacement. Nat Struct Mol Biol 2007, 14:540-547.

26. Dechassa ML, Zhang B, Horowitz-Scherer R, Persinger J, Woodcock CL, Peterson CL, Bartholomew B: Architecture of the SWI/SNF-nucleosome complex. Mol Cell Biol 2008, 28:6010-6021.

27. Gat-Viks I, Meller R, Kupiec M, Shamir R: Understanding gene sequence variation in the context of transcription regulation in yeast. PLOS Genet 2010, 6:e1000800

28. Kenneth NS, Mudie S, van Uden P, Rocha S: SWI/SNF regulates the cellular response to hypoxia. J Biol Chem 2009, 284:4123-4131

29. Johnson $A B$, Barton MC: Hypoxia-induced and stress-specific changes in chromatin structure and function. Mutat Res 2007, 618:149-162.

30. Huh WK, Falvo JV, Gerke LC, Carroll AS, Howson RW, Weissman JS, O'Shea EK: Global analysis of protein localization in budding yeast. Nature 2003, 425:686-691.

31. Hon T, Dodd A, Dirmeier R, Gorman N, Sinclair PR, Zhang L, Poyton RO: A Mechanism of Oxygen Sensing in Yeast: Multiple Oxygen-Responsive Steps in the Heme Biosynthetic Pathway Affect Hap1 activity. J. Biol. Chem 2003, 278:50771-50780.

32. Shulga N, Roberts P, Gu Z, Spitz L, Tabb MM, Nomura M, Goldfarb DS: In vivo nuclear transport kinetics in Saccharomyces cerevisiae: a role for heat shock protein 70 during targeting and translocation. J Cell Biol 1996, 135:329-339.

33. Fahrenkrog B, Hubner W, Mandinova A, Pante N, Keller W, Aebi U: The yeast nucleoporin Nup53p specifically interacts with Nic96p and is directly involved in nuclear protein import. Mol Biol Cell 2000, 11:3885-3896.

34. Leslie DM, Timney B, Rout MP, Aitchison JD: Studying nuclear protein import in yeast. Methods 2006, 39:291-308.

35. Zhang L, Hach A, Wang C: Molecular Mechanism Governing Heme Signaling in Yeast: a Higher-Order Complex Mediates Heme Regulation of the Transcriptional Activator HAP1. Mol Cell Biol 1998, 18:3819-3828.

doi:10.1186/2045-3701-2-30

Cite this article as: Dastidar et al:: The nuclear localization of SWI/SNF proteins is subjected to oxygen regulation. Cell \& Bioscience 2012 2:30

\section{Submit your next manuscript to BioMed Central and take full advantage of:}

- Convenient online submission

- Thorough peer review

- No space constraints or color figure charges

- Immediate publication on acceptance

- Inclusion in PubMed, CAS, Scopus and Google Scholar

- Research which is freely available for redistribution 